\title{
Correction \\ Correction: Critical care management and outcome of severe Pneumocystis pneumonia in patients with and without HIV infection
}

\author{
Xavier Monnet ${ }^{1,2}$, Emmanuelle Vidal-Petiot ${ }^{1,2}$, David Osman ${ }^{1,2}$, Olfa Hamzaoui ${ }^{1,2}$, \\ Antoine Durrbach ${ }^{3}$, Cécile Goujard ${ }^{4,5}$, Corinne Miceli5,6, Patrice Bourée ${ }^{2,7}$ \\ and Christian Richard ${ }^{1,2}$
}

\begin{abstract}
${ }^{1}$ AP-HP, Hôpital de Bicêtre, service de réanimation médicale, 78, rue du Général Leclerc, Le Kremlin-Bicêtre, F-94270, France ${ }^{2}$ Univ Paris-Sud, Faculté de médecine Paris-Sud, EA 4046, 78, rue du Général Leclerc, Le Kremlin-Bicêtre, F-94270, France ${ }^{3}$ AP-HP, Hôpital de Bicêtre, service de néphrologie, 78, rue du Général Leclerc, Le Kremlin-Bicêtre, F-94270, France

${ }^{4}$ AP-HP, Hôpital de Bicêtre, service de médecine interne, 78, rue du Général Leclerc, Le Kremlin-Bicêtre, F-94270, France

5Univ Paris-Sud, INSERM, UMR S 802, 78, rue du Général Leclerc, Le Kremlin Bicêtre, F-94270, France

${ }^{6}$ AP-HP, Hôpital de Bicêtre, service de rhumatologie, 78, rue du Général Leclerc, Le Kremlin-Bicêtre, F-94270, France

${ }^{7}$ AP-HP, Hôpital de Bicêtre, unité des maladies parasitaires, 78, rue du Général Leclerc, Le Kremlin-Bicêtre, F-94270, France
\end{abstract}

Corresponding author: Xavier Monnet, xavier.monnet@bct.aphp.fr

Published: 30 April 2009

Critical Care 2009, 13:407 (doi:10.1186/cc7796)

This article is online at http://ccforum.com/content/13/2/407

(c) 2009 BioMed Central Ltd

See related research by Monnet et al., http://ccforum.com/content/12/1/R28

Following the publication of our article [1], it has been noted that there were two errors in the paragraph under the Results section with the sub-heading $P$. jiroveci pneumonia diagnosis.

The percentage given for positive staining in HIV-negative cases should read $58 \%$, and should be $86 \%$ for HIV-positive cases. This paragraph should read:

"Staining performed on broncho-alveolar lavage specimens was positive in $58 \%$ of HIV-negative cases and in $86 \%$ of HIV-positive cases $(P=0.46)$ (Table 2)."

This paragraph should therefore appear as shown in this correction.

\section{Competing interests}

The authors declare that they have no competing interests.

\section{References}

1. Monnet X, Vidal-Petiot E, Osman D, Hamzaoui O, Durrbach A, Goujard C, Miceli C, Bourée P, Richard C: Critical care management and outcome of severe Pneumocystis pneumonia in patients with and without HIV infection. Crit Care 2008, 12: R28. 